\title{
Heat pipes as perspective base elements of heat recovery in heat supply and ventilating systems
}

\author{
Andrey Matveev ${ }^{1}$, Danila Zelentsov ${ }^{1, *}$, and Alexander Louks ${ }^{1}$ \\ ${ }^{1}$ Samara Technical University, Institute of Architecture and Civil Engineering, Molodogvardeyskaya \\ St., 194, Samara, 443001, Russia
}

\begin{abstract}
Thermotechnical characteristics of heat pipes are considered as high-efficient heat-transfer devices, which can provide energy-saving technologies for heat supply and ventilating systems and for different branches of industry. Thermotechnical and working ("performance capability") characteristics of heat pipes are investigated. By "performance capability" of heat pipes and heat-transfer devices on heat pipes we mean the system state, where it can perform set functions and keep parameter values (thermal power, conductivity, thermal resistance, heat-transfer coefficient, temperature level and differential, etc.) within the regulations of standardized specifications. The article presents theoretical and experimental methods of "gaslock» length determination on noncondensable gases during long-lasting tests of ammonia heat pipes made of aluminum shape AS - KRA 7.5 - R1 (alloy AD - 31). The paper gives results of research of thermotechnical characteristics of heat pipes in horizontal and vertical states (separate and as a set part) while using different systems of thermal insulation. The obtained results of thermotechnical and resource tests show the advantages of ammonia heat pipes as basic elements for heat exchanger design in heating and ventilation systems.
\end{abstract}

\section{Introduction}

Heat supply and ventilating systems of civil and industrial buildings are the main consumers of energy resources. Every year the share of costs is getting higher in budgets of such big cities as Samara. Because of constantly increasing rates of energy sources at present, regeneration of wasted heat of machinery at plants and factures, in communal buildings and residential units is getting more and more attention. Different ways of heat recovery of wasted head are suggested for space heating, its re-usage in heat engine cycles with different design of thermal pumps, heat accumulators, including heat transfer devices of heat pipes [1-5]. The research presents investigation of heat pipes as perspective basic elements for heat exchange in heating and ventilation systems in housing and utility and industrial sectors.

\footnotetext{
* Corresponding author: dvzelentsov@mail.ru
} 


\section{Signs}

\begin{tabular}{|c|c|}
\hline$\Delta V_{\Sigma}$ & total volume of noncondensable gas in the heat pipe after external factors impact \\
\hline$\Delta V_{0}$ & initial volume of noncondensable gases in the heat pipe \\
\hline$\Delta V_{T}$ & volumetric gain of noncondensable gas in the heat pipe after impact \\
\hline$\Delta V_{D}$ & volumetric gain of noncondensable gas in the heat pipe after radiation impact \\
\hline$K$ & the constant of reaction rate of ammonia thermal decomposition \\
\hline$\tau$ & the duration of thermal impact \\
\hline$V_{0}$ & initial ammonia volume in the heat pipe \\
\hline$K_{0}$ & preexponential factor \\
\hline$E_{\text {act }}$ & molar heat of thermo chemical ammonia decomposition reaction \\
\hline$T$ & temperature \\
\hline$R$ & universal gas constant \\
\hline $\begin{array}{l}\Delta V_{i} \\
\text { end of }\end{array}$ & $\begin{array}{l}\text { volume of } i \text {-mixture component of noncondensable gases in the heat pipe at the } \\
\text { ation period }\end{array}$ \\
\hline $\begin{array}{l}K_{i} \\
\text { decom }\end{array}$ & $\begin{array}{l}\text { the constant on-rate of } i \text {-mixture component at radiation-chemical ammonia } \\
\text { ition }\end{array}$ \\
\hline $\begin{array}{l}a_{i} \\
\text { envirol }\end{array}$ & $\begin{array}{l}\text { corrective coefficient, considering the influence temperature factor of external } \\
\text { nt }\end{array}$ \\
\hline $\begin{array}{l}D \\
\text { consid }\end{array}$ & $\begin{array}{l}\text { radiation rate consumed by heat transfer medium of the heat pipe over a } \\
\text { period of time }\end{array}$ \\
\hline $\begin{array}{l}m_{i} \\
\Delta V_{i}^{T} \\
V_{i}^{0} \\
\text { transf }\end{array}$ & $\begin{array}{l}\text { corrective coefficient, considering the influence of heat pipe body material } \\
\text { volume gain of } i \text {-mixture component resulted from the thermal impact } \\
\text { volume } i \text {-mixture component at the time of filling the heat pipe with heat } \\
\text { edium }\end{array}$ \\
\hline$V_{G L}$ & «gaslock» volume \\
\hline$N_{N C G}$ & amount of substance of noncondensable gases in the heat pipe \\
\hline$p_{\mathrm{NH}_{3}}^{n}$ & saturation pressure of heat transfer medium \\
\hline & area length of noncondensable gases in the heat pipe after pесурсных tests \\
\hline
\end{tabular}

\section{Materials and methods}

Heat pipes (HP) as high-efficient heat transfer devices operate as closed-loop evaporatorcondensing cycle. They are getting more and more applicable in the heat supply and ventilating systems and in different branches of industry. In the simplest case, the heat pipe (fig. 1) has a sealed enclosure, the internal surface of which is covered with a layer of capillary-porous material - wick - saturated with liquid phase of a heat carrier. Different porous materials (grids, porous structures, grooves on the inner surface of enclosure pipes, i.e. any structure that can transfer liquid from the condensation zone into the heating zone due to the action of capillary forces) can be used as a wick (see Fig. 1). 


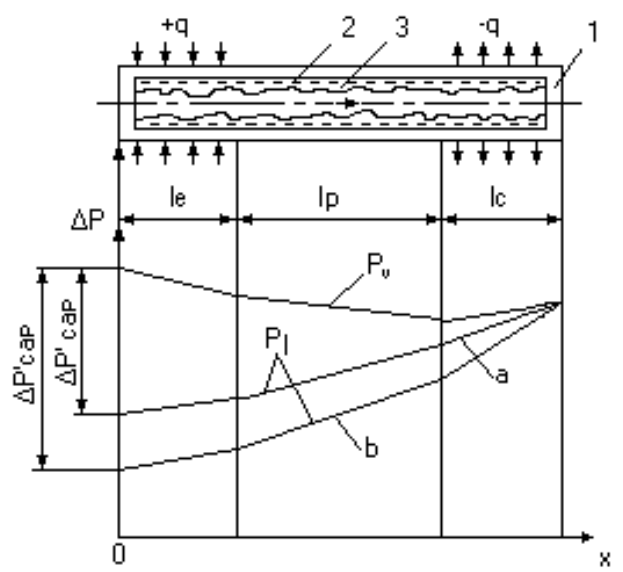

Fig. 1. Diagram of a cylindrical heat pipe and qualitative pressure distribution in a pair of $P_{v}$ and fluid $P_{l}$ without affecting mass forces (a) and in the gravity force field directed against the fluid flow in the wick (b): 1 - body of the heat pipe; 2 - wick structure; 3 - heat carrier; $\pm q$ - heat flow; $l_{e}-$ evaporation zone; $l_{p}$ - transport zone; $l_{c}$ - condensation zone

High heat transfer coefficients in them are explained by the heat transfer of phase transition special intermediate super clean heat transfer device from the heat source to a consumer. For effective performance of separate heat pipes as well as heat pipe system being a part of heat transfer device, for example, hydrodynamic and thermodynamic conditions must be fulfilled. Hydrodynamic conditions characterize the equality of moving forces (capillary, gravitational, etc.) with friction forces, determining pressure losses of vapor and liquid in phase transitions. Thermodynamic conditions are specified by temperature differentials of different parts of heat pipes. Their sum shouldn't exceed the given difference between heat source temperature and its consumer. These are the equations that characterize performance capability of HP. By "performance capability" of heat pipes and heat-transfer devices on heat pipes we will mean the system state, where it can perform set functions and keep parameter values (thermal power, conductivity, thermal resistance, heat-transfer coefficient, temperature level and differential, etc.) within the regulations of standardized specifications.

In the process of using heat pipes in operating heat transfer device, capillary wick structure and inner surface are exposed to different complex physical-chemical reactions. They lead to a solid phase, precipitation and noncondensable gas (hydrogen). The latter is gradually accumulated at the end of condensation area and considerably change the initial thermal technical parameters of HP (thermal power, conductivity, thermal resistance, heattransfer coefficient, etc.). These processes on-rate differ from each other. It is determined by the interaction between operating heat transfer device and porous wick structure and tube wall material, as well as by thermodynamic characteristics of these elementsa. The increase of operating temperature in the pipe considerably intensifies these processes not changing their nature. It gives an opportunity to simulate the rate of noncondensable gas and precipitate depending on the temperature and time and make a forecast about the whole period of HP operation if there isn 't enough time and conditions for testing [6]. The period of operating service under the real conditions must be at least 12 years. It gives an opportunity to compensate the given expenses of HP being a part of heat transfer devices for housing and communal services and industry.

The calculation of gas release and gas lock length of noncondensable gases (NCG) is based upon the data on ammonia $\left(\mathrm{NH}_{3}\right)$ radiation-chemical resistance and its thermal resistance, as well as on laws of ideal gases and a well-known Arrhenius equation $[1,7,8]$. 
Then the general formula of functional relation of NCG volume change from external impact factors for heat transfer devices will be:

$$
\Delta V_{\Sigma}=V_{o}+\Delta V_{T}+\Delta V_{D}
$$

NCG volume gain under thermal ammonia decomposition is calculated by

$$
\Delta V_{T}=V_{O} K \tau
$$

The constant of reaction rate $\mathrm{K}$ is calculated by Arrhenius equation

$$
K=K_{O} \exp \left(-\frac{E_{a c t}}{R T}\right)
$$

Поправочные coefficients, considering temperature influence and construction materials of HP body on on-rate ammonia decomposition and NCG formation are estimated for each of the mixture components [9]:

$$
\Delta V_{i}=k_{i} a_{i} D^{m_{i}}+\Delta V_{i}^{T}+V_{i}^{O}
$$

To calculate the length of gas lock, determining a passive area of condensation, a model of модель «plain front» is taken. According to the model, there is no inter-diffusion of NCG molecules and ammonia vapor at the end of condensation area, and gas lock is an ideal elastic solid, which is acted upon the laws of ideal gases. The volume of NCG gas lock at the end of HT condensation area has been calculated by Mendeleyev-Clapeyron equation

$$
V_{G L}=\frac{n_{N C G} R T}{p_{N H_{3}}^{n}}
$$

Then the gas lock length can be found by the formula given below, where a crosssection ares $S_{S}$ of HP steam channel, $\mathrm{m}^{2}$ (for the testing set of HP made of aluminum shape AS-KRA7.5-R1) is $0.52 \cdot 10^{-4} \mathrm{~m}^{2}$.

$$
L_{G L}=\frac{V_{G L}}{S_{S}}
$$

So the gas lock length can be determined theoretically as well as experimentally.

Experimental research was conducted using curvilinear heat pipes made of aluminum shape AS-KRA7.5-R1 with a flange width of $30 \mathrm{~mm}$, height $-14 \mathrm{~mm}$ and length $L=1762$ $\mathrm{mm}$, bend radius $r=1000 \mathrm{~mm}$, mass $0,63 \mathrm{~kg}$ [12]. Figure 2 shows an experimental installation design. Heat pipes are mounted vertically with a heater down. HP were isolated by $40 \mathrm{Mm}$ foam layers. Heater operation of $20 \mathrm{Om}$ resistance and $600 \mathrm{~mm}$ length was performed with of linear power supply with a voltage of AIP B5.30/10, which has high stability parameters and a low level of noise and pulsations and automatic 2-speed cooling system for reducing vent noise. Measurement and temperature record along HP length is done with a universal measuring instruments like R4833. 


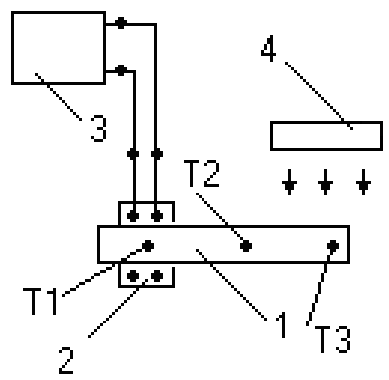

Fig. 2. An experimental installation design with a horizontal position of the heat pipe: 1 - body of a heat pipe; 2 - ohmic heater; 3 - power supply; 4 - cooler; T1, T2, T3 - resistance thermometers for temperature measuring of the pipe wall

In horizontal position pipes have been working as ordinary heat pipes (under the action of capillary forces), and in vertical position (vaporizer at the bottom) - as two-phase thermal siphons (under the action of gravitational force). Positive angle in relevance to horizon (including vertical HP position) at the process of NCG determination is chosen only for elimination of possibility of capillary structure draught and the influence of excess amount of heat transfer device on HP characteristics. When placed horizontally, the excess of liquid heat transfer medium $(\sim 10 \%)$, as well as NCG, are expelled by water-vapor saturation pressure at the end of condensation area, which leads to a difficulty with control of the NCG absence in HP and HP quality control. In vertical position the liquid excess is concentrated at the HP bottom in the form of a "puddle" and has no influence on NCG gas escape. Acceleration of degradation processes of characteristics of heat pipes is achieved because of their operation at higher temperature (up to critical ammonia temperature). Defect feature will be a higher length increase of blocked gases area compared to a standard quality or penetrability of HP because of corrosion. But at the beginning of endurance tests they should be considered as HP of constant conductivity, and when the endurance test time is increased and there is NCG accumulation at the end of condensation area (when the NCG length area occupies a part of active ammonia condensation vapor) - heat pipe of alternating conductivity (and alternating thermal resistance).

\section{Results}

The result analysis of tests [9-11] shows, что maximum transferred thermal power of HP (AS-KRA7.5-R1) is approximately $250 \mathrm{~W}$ at $25{ }^{\circ} \mathrm{C}$ saturation temperature at transportation zone. The main HP thermal technical characteristics after 971-hour operation in vertical and horizontal positions conditions thermal conditions: $Q=15 \mathrm{~W}$ and external environment temperature of $18{ }^{\circ} \mathrm{C}$ temperature in evaporation and condensation areas are correspondingly $T 1=24.22 \div 24.39{ }^{\circ} \mathrm{C}, T 2=23.68 \div 23.88{ }^{\circ} \mathrm{C}$, temperature differential along HP length $\Delta T=0.54 \div 0.51{ }^{\circ} \mathrm{C}$, thermal conductivity $k F=Q / \Delta T=27.78 \div 29.41 \mathrm{~W} / \mathrm{K}$, thermal resistance $R=\Delta T / Q=0.036 \div 0.034 \quad \mathrm{~K} / \mathrm{W}$, heat transfer coefficient $k=Q /(F \Delta T)=180554 \div 191148 \mathrm{~W} /\left(\mathrm{m}^{2} \cdot \mathrm{K}\right)$. After 1210 -hour operation of HP the main thermal technical HP characteristics practically remain the same. Pipes of AS-KRA7.5-R1 with a $\Omega$ shaped grooved wick structure are characterized by heat conductivity and low thermal resistance. It allows constructors to use them as basic elements of heat transfer apparatus in heat-supply and ventilating systems, boilers, etc. In experiments with a system of heat pipes and constant temperature of $85 \pm 2{ }^{\circ} \mathrm{C}$ of the body, the power supply considering mutual influence of heat interaction fluctuates between $20 \div 33 \mathrm{~W}$, and in case of separate location and thermal isolation $-20 \div 23 \mathrm{~W}$. 


\section{Discussion}

The heat appliance as a heat radiator of HP should also be considered and worked upon. It is necessary to decide how the flow-pipes are distributed - from two-phase thermal siphon to radiator of HP; how HP radiator is connected with supplied flow pipes and its thermal regulation. Heat exchange units of HP are also efficient in cases of heat recovery of vent emissions and the usage of flue gases heat for satisfying the needs in heat water and warm air [2]. Particularly, the device with extended surface heat pipes was produced. The vaporizers extended surface heat pipes have been located in the flow of warm wasted air, and a capacitor - in the cool air flow to pre-heat the latter one. Efficiency coefficient of such a regenerative heat exchanger is $70 \%$, with a payoff period of about 2 years. Heat exchange units on ammonia HP like air - air are advisable to place on the upper technical floors of civil and communal buildings.

\section{Conclusions}

The undertaken studies show that heat exchangers of heat pipes are able to work for a long time keeping given parameters (thermal capacity, conductivity, heat-transfer resistance, heat transfer index, thermal gradient, etc.) within the limits prescribed by norms and specifications. The obtained results of thermotechnical and resource tests prove the advantages of ammonia heat pipes as basic elements for heat exchanger design in heating and ventilation systems. The further studies aim at design of heat exchangers constructions, at their package treatment and implementation.

\section{References}

1. P.D. Dan, D.A. Ray, Heat pipes: transl. from English (Energy, Moscow, 1979)

2. D.V. Zelentsov, A.L. Louks, Traditions and innovations in civil engineering. Construction technologies, 315-317 (2015)

3. V.P. Frolov,S. N.S. Sherbakov, M.V. Frolov, A.Y. Tshelginskiy, Energy conservation 2, 50-53 (2004)

4. V.P. Frolov, N.S. Sherbakov, M.V. Frolov, A.Y. Tshelginskiy, Heat Supply Messenger 7, 34-39 (2004)

5. L.L. Vasiliev, V.G.Kiselev, Y.N. Matveyev, F.F. Molodkin, Heat exchange - recovery units on heat pipes (Science and technology, Minsk, 1987)

6. A.L. Louks, E.A. Krestin, A.G. Matveev, A.V. Shabanova, A.I. Kitaev, Urban Construction and Architecture 1 (22), 34-40 (2016). doi: 10.17673/Vestnik.2016.01.6

7. M.N. Ivanovsky, V.P. Sorokin I.V. Yagodkin, Physics of heat pipes (Atomizdat, Moscow, 1978)

8. L.L. Vasiliev, Heat exchange units of heat pipes (Science and technology, Moscow, 1981)

9. A.L. Louks, E.A. Krestin, A.G. Matveev, V.I. Vesnin, Urban Construction and Architecture 3 (24), 17-24 (2016). doi: 10.17673/Vestnik.2016.03.3

10. SFBC. 067314.009PP3, Heat Pipe. Parameters calculation for accelerated endurance tests (USP STC «Thermalenergytechnics», Protvino, 1998)

11. A.L. Louks, A.G. Matveev, Messenger of SSU 6(56), 401-418, (2007) 